\title{
THE COMPLETE PATTERN OF OCULAR DOMINANCE STRIPES IN THE STRIATE CORTEX AND VISUAL FIELD OF THE MACAQUE MONKEY ${ }^{1}$
}

\author{
S. LEVAY,$^{* 2}$ M. CONNOLLY $\ddagger^{3}$ J. HOUDE $\ddagger$ AND D. C. VAN ESSEN $\ddagger$ \\ * Department of Neurobiology, Harvard Medical School, Boston, Massachusetts 02115, and $\ddagger$ Division of Biology, California \\ Institute of Technology, Pasadena, California 91125
}

Received April 26, 1984; Accepted July 19, 1984

\begin{abstract}
Ocular dominance stripes in the striate cortex of a macaque monkey were labeled by autoradiography after injection of $\left[{ }^{3} \mathrm{H}\right]$ proline into one eye. The stripes were reconstructed on a representation of the flattened cortical surface by two independent techniques: one used computer graphics, and the other was the manual unfolding procedure of Van Essen and Maunsell (VanEssen, D. C., and J. H. R. Maunsell (1980) J. Comp. Neurol. 191: 255-281). The two reconstructions differed in many details of the pattern but were in agreement on its general features. As described in earlier studies, the stripes formed a system of parallel bands, with numerous branches and islands. They were roughly orthogonal to the V1/V2 border throughout the binocular segment of the cortex. In the lateral part of the operculum, where the fovea is represented, the stripes were less orderly than elsewhere. In the calcarine fissure the stripes ran directly across the striate cortex from its dorsal to its ventral margin. In the far periphery the stripes for the ipsilateral eye became progressively narrower, eventually fragmenting into small islands at the edge of the monocular segment. The overall periodicity (width of a left-plus right-eye pair of stripes) averaged $0.88 \mathrm{~mm}$ but decreased by a factor of about 2 from center to periphery. This decrease was not accounted for solely by shrinkage of the ipsilateral eye stripes.

The flattened cortical reconstruction was transformed back into visual field coordinates, using information about visual field topography obtained from the detailed mapping study of Van Essen et al. (Van Essen, D. C., W. T. Newsome, and J. H. R. Maunsell (1984) Vision Res. 24: 429-448), as well as from more limited mapping done in the same monkey that was used for the reconstruction. In the transformed map, the stripes increased in width about 40 -fold from the fovea to the far periphery. As deduced previously (LeVay, S., D. H. Hubel, and T. N. Wiesel (1975) J. Comp. Neurol. 159: 559-576; Hubel, D. H., and D. C., Freeman (1977) Brain Res. 122: $336-343)$, there were portions of the map in which the stripes followed curves approximating isoeccentricity lines, but this relationship was not very exact or consistent. The pattern of stripes appears to be more meaningfully related to the geometry of the cortical surface. This has significant implications for understanding the developmental mechanisms involved in stripe formation.
\end{abstract}

The striate cortex (area 17, V1) is the first major site on the visual pathway at which information from the two eyes is integrated. In many species, geniculocortical afferents serving the left and right eyes terminate in layer $4 \mathrm{C}$ as alternating bands-ocular dominance stripes. Within a stripe, cells respond only to stimulation of the appropriate eye. By virtue of intracortical projections, which are predominantly vertical, this eye dominates the responses of cells in a slab of tissue extended from pia to white matter-an ocular dominance column (Hubel

\footnotetext{
${ }^{1}$ We thank R. Leibowitz for histological assistance, M. Peloquin for photography, K. Tazumi for illustrations, and C. Oto and N. Goodknight for assistance in preparation of the manuscript. This work was supported by National Institutes of Health Grants EY-R01-01960 (to S. L.) and EY-R01-02091 (to D. C. V. E.)

${ }^{2}$ To whom correspondence should be sent, at his present address: The Salk Institute, P. O. Box 85800, San Diego, CA 92138.

${ }^{3}$ Present address: University of California of Medicine, San Francisco, CA 94143.
}

and Wiesel, 1968). The present study is aimed at achieving a detailed description of the layout of ocular dominance stripes in the striate cortex of the macaque monkey.

Much is already known about the anatomy of ocular dominance stripes in the macaque. In a large part of striate cortex they have a relatively constant width of about $400 \mu \mathrm{m}$ (Hubel and Wiesel, 1972). Seen from the cortical surface, the layer $4 \mathrm{C}$ stripes are parallel and often of indefinite length, but there are numerous branches and isolated segments. There is a general similarity in the pattern of stripes from one animal to the next, at least for the part of the cortex that represents the central $15^{\circ}$ or so of the visual field (LeVay et al., 1975). For example, there is a strong tendency for the stripes to meet the margin of striate cortex (the V1/V2 border) at right angles.

The method best suited for demonstrating the complete layout of ocular dominance columns is the autoradiographic detection of $\left[{ }^{3} \mathrm{H}\right]$ proline transported transneuronally from one eye (Grafstein, 1971; Hubel et al., 1974a). Although the cortex is a highly folded structure, a two-dimensional reconstruction 
of the columns can be obtained, either by manual reconstruction (Van Essen and Maunsell, 1980) or by computerized graphics techniques. We have applied both of these methods in an attempt to answer several questions about the distribution of ocular dominance stripes. First, are there systematic differences, either in their spacing or in the balance between the eyes, in different regions of area 17 ? We have found that there are at least two such differences: a strong dominance of the contralateral eye in the periphery of the visual field, and a significant difference in the average periodicity of the stripes between the representation of the vertical meridian and the far periphery.

Second, what significance should be attached to the actual pattern formed by the stripes on the cortex? One possibility is that some orderly relationship exists between the orientation of the stripes and the topography of the visual field representation. This possibility has previously been explored by Hubel and Freeman (1977). They took the partial reconstruction by LeVay et al. (1975) and transformed it back onto a map of the visual field, using information about field layout and magnification factors available at the time (Daniel and Whitteridge, 1961; Hubel and Wiesel, 1974). They concluded that in much of the cortex the columns ran close to the representation of isoeccentricity lines. By combining partial physiological mapping, autoradiography, and techniques for unfolding the cortex in the same animal, and by reference to recently published detailed field maps (Van Essen et al., 1984), we have been able to make a detailed comparison of the layout of the stripes on the cortex and their layout in the visual field. We find that the orientation of the stripes conforms more closely to the geography of the cortex than to the topography of the visual representation, an observation which may be of significance in understanding the formation of this pattern during development.

\section{Materials and Methods}

The bulk of the results presented in this paper were obtained from a single monkey, a 2-year-old female crab-eating macaque (Macaca fascicularis), weighing $1.9 \mathrm{~kg}$. It received two injections, spaced 4 days apart, of $\left[{ }^{3} \mathrm{H}\right]$ proline into the left eye. Each injection comprised $2 \mathrm{mCi}$ of $2,3-\left[{ }^{3} \mathrm{H}\right.$ |proline (specific activity, 20 to $40 \mathrm{Ci} / \mathrm{mmol}$ ) dissolved in 25 $\mu \mathrm{l}$ of $0.9 \% \mathrm{NaCl}$. The injections were made into the vitreous with a 27 gauge needle attached to a length of polyethylene tubing. Fourteen days after the second injection, the monkey was prepared for acute recording. It was anesthetized initially with ketamine, followed by intravenous pentobarbitol. Gallamine triethiodide was administered to abolish eye movements, and the monkey was mechanically ventilated. Retinal landmarks were plotted onto a tangent screen with a reversingbeam opthalmoscope. Two horizontal penetrations were made with a tungsten microelectrode into the left striate cortex, close to the midline. They were aimed so as to pass through the dorsal and ventral banks of the calcarine fissure, as well as the opercular cortex. Small electrolytic lesions ( $5 \mu \mathrm{A}, 5 \mathrm{sec}$, electrode negative) were placed at the termination of each penetration.

At the end of the recording session the monkey was perfused with $10 \%$ formol saline. The left occipital lobe was divided into two parts with a horizontal knife cut. Each part was sunk in $30 \%$ sucrose and frozen sections were cut in the horizontal plane. The $20-\mu$ m-thick sections were collected five to a vial; one section from each vial was mounted on a gelatinized slide and processed for autoradiography using Kodak N'I'B2 emulsion. After 3 months' exposure, the slides were developed in Kodak D19. Other sections were mounted and stained with cresyl violet for reconstruction of the electrode tracks. The right hemisphere (contralateral to the injected eye) was processed similarly, except that it was sectioned in a plane tangential to the center of the operculum (the plane used by I eVay et al. (1975) in their reconstruction using the Liesegang silver method).

Every autoradiograph was photographed at a low power with darkfield illumination. The photographs were printed at a final magnification of $\times 7.6$ after correction for $16 \%$ tissue shrinkage during histological processing. The set of 203 photographs of the left occipital lobe formed the basis for the reconstruction of the columnar pattern in this lobe.

Two entirely independent methods were used to derive this reconstruction. The first involved computer graphics. Tracings were first made of the labeled patches in layer $4 \mathrm{C}$ for each photograph. Also included in the tracings was the V1/V2 border, wherever it appeared, and any places where the electrode tracks traversed layer 4C. From these a second set of tracings was obtained in which, by judicious rotations and displacements, all mismatches between adjacent sections that had arisen during the mounting of the sections were eliminated. The matching process was performed separately for 10 different segments of striate cortex (operculum, roof of calcarine fissure, and so on). The set of tracings for each segment was then entered in the computer by means of a graphics tablet. Successive sections were aligned with reference to the anterior edge of the block and to the V1/ V2 border where it appeared in the sections; the labeled ocular dominance stripes were not themselves used for alignment. Once entered into the computer, each segment was rotated in three-dimensional space to obtain a surface view and then was photographed. Segments with considerable curvature were photographed more than once: for example, the operculum was photographed from five different angles. From each photograph a tracing was prepared in which the labeled stripes, shown on the computer screen as stacks of line segments, were represented by solid bands. For the most part, this filling-in process presented no difficulty, but in some areas, notably the foveal representation where the stripes are highly fragmented and irregular, there was some ambiguity which had to be resolved subjectively. The tracings were then assembled into a complete reconstruction using the pattern of stripes along the margins of each tracing for purposes of alignment. Even so, there was sometimes ambiguity in the matching of individual stripes across the boundaries between segments. It was also necessary to introduce several relieving cuts around the perimenter of the recon struction. The exact position of these cuts was arbitrary, but they were mainly required at the two ends of the oval reconstruction (near the foveal representation and in the extreme periphery). The outline of this reconstruction, without ocular dominance stripes included, is shown in Figure $1 A$. Dotted lines in Figure $1 A$ denote the major lines of folding that exist in vivo. The locations of the six recording sites are also indicated on the map; receptive field locations for these sites are shown in Figure $1 B$.

The second, manual, method of reconstruction has been described by Van Essen and Maunsell (1980). In brief, tracings were made of layer $4 \mathrm{C}$ contours from sertions taken at $1-\mathrm{mm}$ intervals. These contours were then transferred to a separate sheet, on which they were aligned with respect to one another so as to provide an accurate representation of surface area, with minimal distortion of linear relationships. Figure $1 C$ shows the outline of the resultant cortical map, along with contour lines from sections at $3-\mathrm{mm}$ intervals, i.e., every third contour used in constructing the map. Dotted lines in Figure $1 C$ indicate the medial edge of the operculum and the major folds of the calcarine sulcus. The locations of the six recording sites are also shown. In order to minimize distortions, a single relieving cut was introduced in the anterior portion of striate cortex, near the fundus of the stem of the calcarine sulcus. The magnitudes of the residual distortions in the map were estimated using the procedure described in an earlier study of striate cortex (Van Essen et al., 1984). Twelve different regions of the cortical surface which were judged to be reasonably flat and precisely $2 \mathrm{~mm}$ on a side were marked on the section contours and then transferred to the cortical map, where they are denoted as blackened compartments (Fig. $1 C$ ). Planimetric measurements on these compartments indicate that there is very little distortion of surface area (mean, $8 \%$, maximum, $12 \%$ ). The shear between contours, as measured by the angular distortion of the corners of the compartments, ranged from $0^{\circ}$ to $25^{\circ}$ (mean, $12^{\circ}$ ), corresponding to 0 to $10 \%$ linear distortion.

Once the manual version of the map was completed, the ocular dominance stripes were entered from each of the 203 section photographs. Registration betwen sections was achieved by accurate alignment of various landmarks, such as the points of sharpest curvature along gyri and sulci, as well as by ensuring that there was reasonable alignment of stripes on neighboring sections. Each stripe was initially entered as a line segment; segments from neighboring sections were subsequently filled in to form continuous stripes. As with the computer reconstruction, this process was fairly straightforward, but there were many places in which matches had to be inade subjectively.

The orientation of the maps in Figure 1 deserves comment. We have 


\section{A}
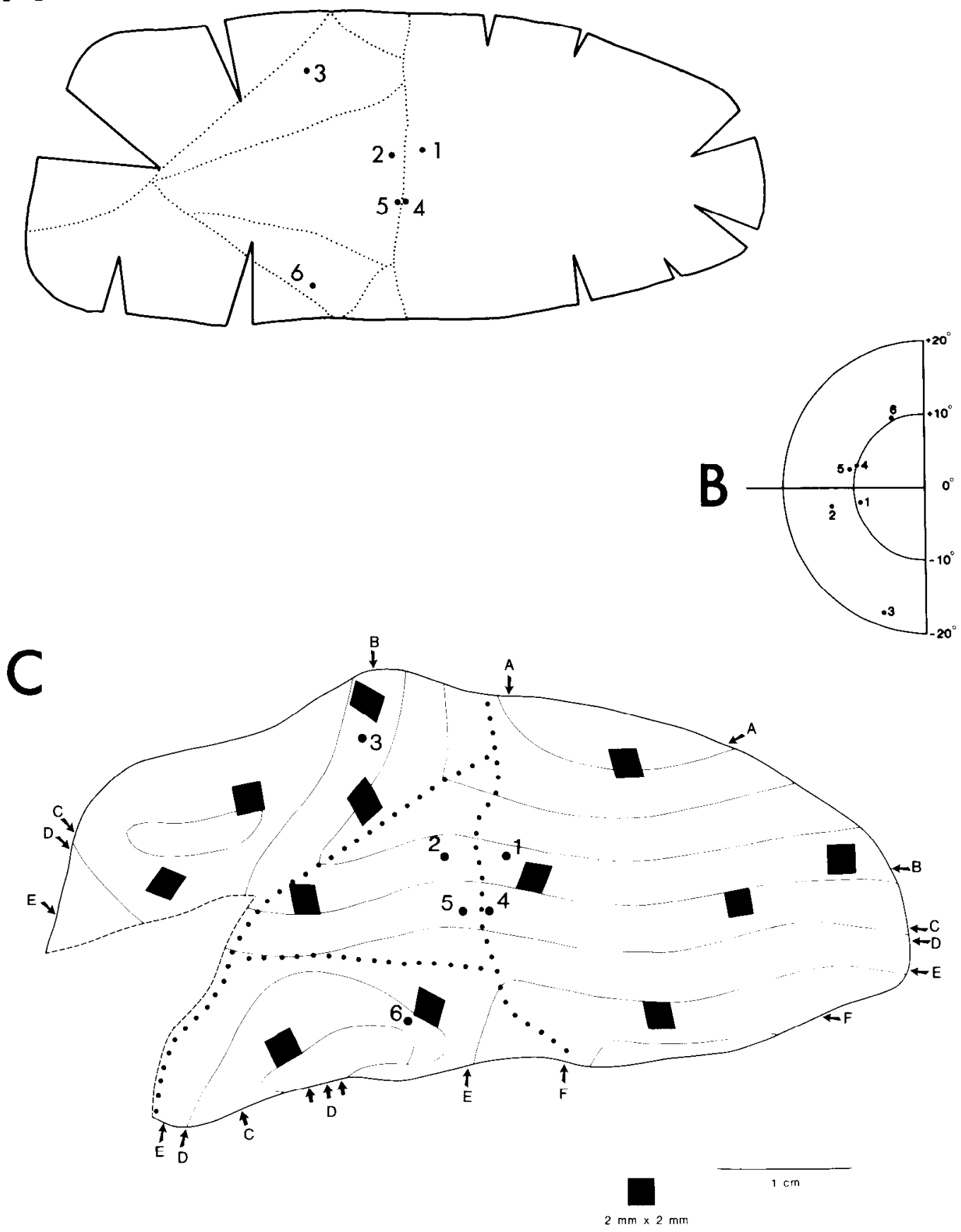

Figure 1. Outlines of the unfolded, flattened striate cortex. A, The reconstruction based on computer graphics. Dotted lines indicate major sectors of striate cortex which were individually reconstructed from face-on viewing angles before being joined. The operculum is represented on the right; various portions of the calcarine sulcus are on the left. Locations of six recording sites made in two microelectrode penetrations are also shown. $B$, Receptive field locations for recording sites 1 to 6 in the striate cortex. $C$, The manual reconstruction. Contour lines are from sections taken at 3-mm intervals. Letters $(A$ to $F$ ) along the margin indicate where the contours of the six sections in Figure $4(A$ to $F$ ) meet the border of the striate cortex. Solid squares ("compartments") denote regions corresponding to squares $2 \mathrm{~mm}$ on a side in the intact hemisphere. Each compartment was identified by choosing a $2-\mathrm{mm}$ segment from a smooth region on one section and identifying a section in which the corresponding segment was $2 \mathrm{~mm}$ away (including components of separation within the plane of section as well as between sections). The locations of these segments were than transferred to the cortical map, and the compartment was filled in. Locations of recording sites are indicated in the same manner as for the preceding map. The separations between sites differ for the two maps, especially for sites 4 and 5 , mainly because of foreshortening along the medial margin of the operculum in the computer map.

adopted the convention that all cortical maps are illustrated as though they were from the right hemisphere, irrespective of their hemisphere of origin. This convention, also used in a number of earlier studies (e.g., Van Essen and Maunsell, 1980; Van Essen et al., 1984), results in the foveal representation of striate cortex always being on the right side of the map and the inferior field representation always on top.

Although they are by no means identical, the two maps of striate cortex are similar in overall size and shape. The surface area of the 
manual map was determined to be $1380 \mathrm{~mm}^{2}$; that of the computer map is $1050 \mathrm{~mm}^{2}$, a difference equal to $24 \%$ of the manual version. This discrepancy is partly attributable to residual foreshortening in various parts of the computer map where compensation for curvature of the cortical surface was incomplete.

Transformation from cortex to visual field. To determine how the ocular dominance stripes would appear if projected onto the visua field, it is necessary to know the relationship between cortical coordinates and visual field coordinates throughout the representation. To determine this relationship, we used information about the cortical magnification factor, as was done by Hubel and Freeman (1977), except that we were able to use recently published data that provide a more accurate and complete analysis of the representation in striate cortex (Van Essen et al., 1984). In this study, it was found that the relationship between magnification and eccentricity can be reasonably approximated by a modified power function of the general form

$$
M_{a}=a(b+E)^{-x}
$$

where $M_{a}$ is the areal magnification factor (square millimeters of cortex per square degree of visual field), $E$ is the eccentricity and $a, b$, and $x$ are adjustable parameters which determine the exact size and shape of the map and the dependence of magnification on eccentricity. The specific values of these parameters were determined for one hemisphere that was mapped in detail ( $a=103, b=0.82, x=2.28$ ), but it was also shown that there is substantial individual variability in the size, shape, and internal organization of striate cortex and hence in the values of all three parameters. In addition, there are anisotropies in some parts of the representation, such that the linear magnification factor (millimeters of cortex per degree of visual field) depends on the direction in which it is measured. These anisotropies are not embodied in the simplified expression for areal magnification shown in equation 1 .

We used computer-generated models that were based on equation 1 (with adjustments for anisotropies) to find appropriate values of the parameters for the actual hemisphere in which the ocular dominance stripes were mapped. The information used in making this fit included the overall size and shape of the cortical map, as well as several pieces of information related to its topographic organization.

The computer algorithm for graphically generating model representations involved several computational stages. First, the visual hemifield was subdivided into 100 hemi-annuli, each representing a narrow eccentricity range. Each hemi-annulus was then divided by radial lines into 60 compartments. The transformation to the cortical representation was carried out in sequential fashion by appropriately magnifying and transposing each compartment. The sequence began at the fovea and continued with a full set of compartments at each eccentricity range before moving on to the next eccentricity range. Each step involved taking a compartment in the visual field, magnifying it according to the amount specified by the parametes being used in conjunction with equation 1 , and positioning it properly in relation to the portion of the cortical map already generated. One of these steps is illustrated schematically in Figure 2, which shows a compartment from the lower visual field and its appropriately magnified representation in the upper half of the partially generated cortical model (the inversion reflecting the known orientation of the visual representation in striate cortex). For clarity, the transformation in Figure 2 is shown for a compartment much larger than those actually used in the algorithm; their actual size is indicated for one small region on each map. A key point in understanding the algorithm is that the compartment under construction starts with three of its corners already determined. The coordinates of the fourth corner are determined on the basis of the total area of the cortical compartment and on the presence of any of the aforementioned anisotropies in the linear magnification factor. The anisotropies in the model are produced by shifting the fourth corner of the compartment by an amount and direction that keeps the area constant while producing the desired ratio of linear magnification factors along lines of constant eccentricity and polar angle (see Figure 2 legend)

The pronounced curvature of the vertical meridian in Figure $2 A$ is an intentional consequence of the procedure we used for representing solid angles in the visual field with minimal distortion. A two-dimensional representation of an intrinsically curved surface (the visual field or the retina) cannot be made without some type of distortion. The algorithm we chose for mapping the visual hemifield is one which allows a modest degree of shear (angular distortion) at high eccentric- ities in exchange for no discontinuities and no stretching (areal distortion).

Further details of the mapping algorithm will be described elsewhere (D. C. Van Essen and J. Houde, unpublished results). For the present purposes, it is sufficient to illustrate the adequacy of the technique by showing the final result, a model map, in comparison to the map from the actual experimental hemisphere. Figure 3 shows the outlines of both maps, along with several markers of visual topography. In particular, the map of the experimental hemisphere (Fig. 3A) shows the horizontal meridian and $10^{\circ}$ isoeccentricity line, determined from receptive fields of nearby recording sites, plus the optic disc and monocular crescent representations determined from the autoradiographic results following the eye injection. The model map (Fig. $3 B$ ) shows the corresponding topographic coordinates. Obviously, the two maps are not identical, as there were limitations in the degree to which the computer algorithm could replicate features such as the curved shape of the $10^{\circ}$ isoeccentricity line (see Figure 3 legend). The actual parameters of the expression for areal magnification selected for the bestfitting model are given in equation '2 as

$$
M_{a}=100(0.8+E)^{-2.0}
$$

The resultant model of striate cortex was encoded in computer memory as a set of coordinates for the vertices of all 6000 compartments in the visual fields ( $x, y$ space) and a set of coordinates for the corresponding compartments in the cortex ( $u, v$ space). These data arrays formed the basis for projecting ocular dominance stripes onto the visual field. The first step was to enter the outlines of all stripes onto computer disk memory using a grpahics tablet. (The minimum spacing between data points correspond to $0.3 \mathrm{~mm}$ in the cortex, and the complete set of stripes was represented by approximately $10^{4}$ points.) A separate computer program treated each data point from the ocular dominance outline as a pair of $u, v$ coordinates, identified the cortical compartment within which the point was contained, and determined the location of the point relative to the vertices of the compartment. The point was then assigned visual field $(x, y)$ coordinates on the basis of this information about its location within an identified compartment. Points belonging to the same outline were connected together, and the resultant back-transformed stripes were displayed on a graphics plotter. Because of the wide range ( 50 -fold) in dimensions of the back-transformed stripes, it was necessary to make several partial plots at different magnifications and then combine the images after appropriate photographic enlargement.

Other hemispheres. The right hemisphere from the main experimental animal was sectioned tangential to the opercular cortex, and photographic montages were prepared of the pattern of stripes over much of the operculum and the roof of the calcarine fissure. These montages are in effect planar projections, and to avoid marked distortions they were not extended to regions of high curvature.

Besides the one monkey used for the detailed reconstruction, several other monkeys that had received eye injections of $\left[{ }^{3} \mathrm{H}\right]$ proline were studied. In order to confirm our observations about the layout of ocular dominance stripes in the representation of the peripheral visual field, photographic montages of the labeling pattern in the roof of the calcarine fissure were prepared from one of these monkeys.

\section{Results}

Perhaps on account of the double injection, the transneuronal labeling was unusually strong in both occipital lobes. As is usually the case, labeling was heaviest in the representation of the far periphery of the visual field and weaker centrally, but even at the representation of the fovea stripes, the stripes were clearly recognizable. The regional differences in density of label may be related to the fact that there is greater emphasis on central vision in striate cortex than at the retinal ganglion cell level (Rolls and Cowey, 1970; Malpeli and Baker, 1975; Van Essen et al., 1984). Hence, the number of cortical cells to which each retinal ganglion cell relays its outputs must be larger for central than for peripheral vision.

Examples of six sections from the horizontally sectioned set of autoradiographs of the left hemisphere (ipsilateral to the injected eye) are shown in Figure 4. Simple inspection of the autoradiographs suggested some surprising differences in the appearance of the stripes in different parts of the cortex. For 


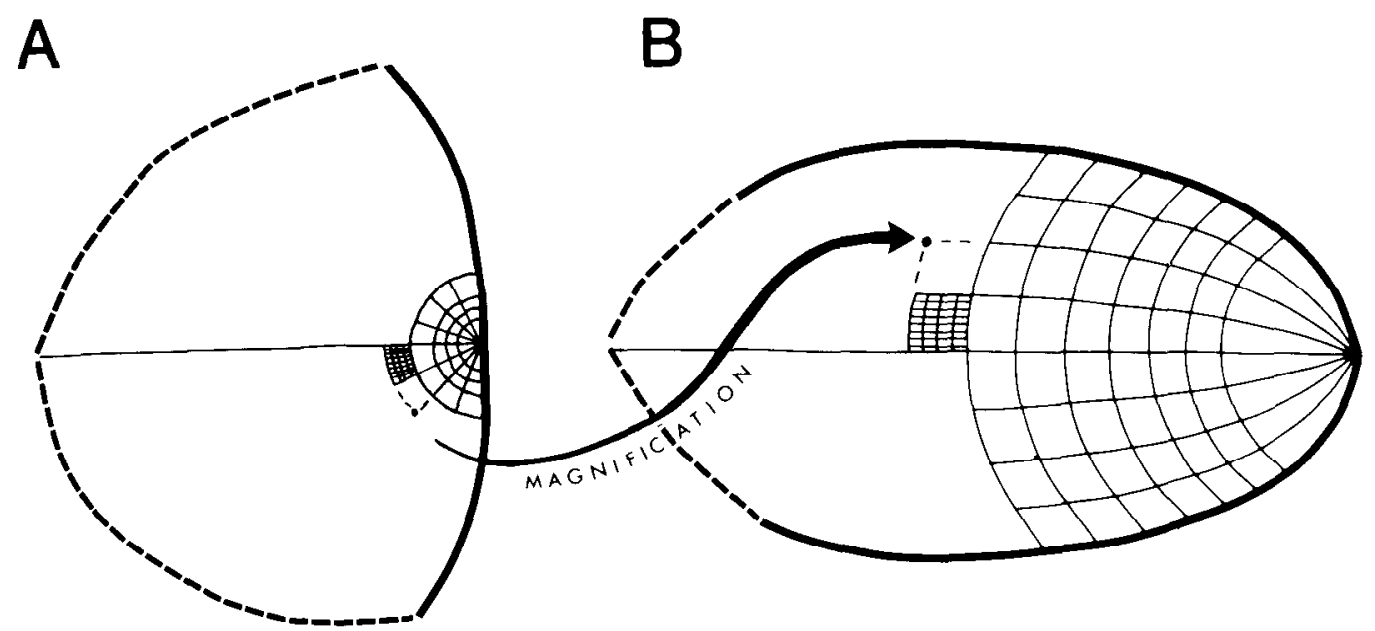

\section{VISUAL FIELD}

\section{CORTICAL MODEL}

Figure 2. A schematic diagram of how mathematical models of the visual representation in striate cortex were generated. In this illustration, each compartment in the visual field $(A)$ is magnified according to the equation $M_{\mathrm{a}} \alpha(1+\mathrm{E})^{-2}$, with no anisotropies or dependence of magnification of polar angle. In the more general case, several types of anisotropy could be introduced. One form of anistropy was a horizontal "shearing" between contours that produced an overall tilt of isoeccentricity lines in a direction and magnitude specified by one of the input parameters. Another anisotropy was a "bending' of isoeccentricity contours that reduced or even reversed their rightward curvature. To prevent oscillations or other breakdowns of the algorithm, it was necessary to start with only minimal shear or bending for the first few contours (nearzero eccentricity) and to build up gradually to the desired effect at higher eccentricities. For reasons such as these, the exact mathematical formulation for introducing shear was cumbersome and will not be detailed here. The essential points are: (1) that the actual magnitude of shearing and bending can best be assessed by inspecting the graphically displayed cortical model $(B)$ and $(2)$ that all of the shearing and bending was done in such a way that the areal magnification was unaffected.

example, the stripes seemed to be more closely spaced in the stem of the calcarine fissure, where the far periphery is represented (arrows in Fig. 4, $D$ and $E$ ) than in other parts of the cortex. Also, there were regions where the labeled patches were much narrower than the gaps between them (arrow in Fig. 4C), suggesting an imbalance in the size of ocular dominance stripes for the two eyes. It was hard to tell, however, just by inspecting the autoradiographs, whether these were true regional variations or merely the result of differences in the regularity of the pattern of stripes or in their orientation with respect to the section plane.

There were two regions in which the labeling was continuous rather than patchy. One was a small, heavily labeled zone in the roof of the calcarine fissure (Fig. $4 B$ ). This was the representation of the optic disc in the contralateral (noninjected) eye. The other was a larger, weakly labeled area adjoining the border of striate cortex far anterior on the dorsal bank of the calcarine fissure ( $\mathrm{Fig}$. 4, $\mathrm{C}^{\prime}$ to $E^{\prime}$ ). 'This was the representation of the contralateral eye's monocular crescent. The presence of some label in this region may be attributed to leakage of label across layers in the lateral geniculate nucleus (LGN) (LeVay et al., 1978).

The labeling pattern in the entire set of sections of the left hemisphere was entered into the computer (see "Materials and Methods"). This was done separately for 10 different segments of striate cortex. The reconstructions of three of these (the operculum, the roof of the calcarine fissure, and the dorsal bank of the stem of the fissure) are shown as stereo pairs in Figure 5. In the figure, each segment has been rotated by the computer to give an en face view of the columnar pattern. The opercular segment (Fig. $5 \mathrm{~A}$ ) shows a pattern similar to one that was prepared previously with the Liesegang silver method (LeVay et al., 1975). When viewed with stereo glasses, the pattern is seen to be foreshortened around the edges, where the cortex curves away from the viewer. For this reason, a total of five different views of the opercular segment were photographed. The roof of the calcarine fissure (Fig. $5 B$ ) is more nearly flat. It has two striking features: an elongated region of continuous label, which is the representation of the optic disc, and a region lateral to the optic disc where the labeled bands (ipsilateral eye stripes) are clearly much narrower than the unlabeled gaps (contralateral eye stripes.) The dorsal bank of the stem of the fissure (Fig. $5 \mathrm{C}$ ) contains a region of relatively narrowly spaced stripes, close to an unlabeled zone which is part of the monocular crescent representation (i.e., the zone receiving input only from the contralateral eye).

The surface of the striate cortex contains a significant amount of intrinsic curvature (i.e., curvature remaining after unfolding). In Figure 5, $A$ and $B$, it can be seen that the sign of the intrinsic curvature is not constant, but rather is pia-outward for the operculum and pia-inward for the roof of the calcarine sulcus. After complete unfolding, but with no tearing, stretching, or shearing, such a surface would have one mound-like elevated region adjacent to a moderately depressed region, rather than a simple bathtub-like appearance. This has been confirmed using a latex sheet cast over a scale model of a macaque hemisphere (D. C. Van Essen, unpublished observations). The existence of this intrinsic curvature is what necessitated the discontinuities at several places along the margins of the computer map and the single discontinuity (coupled with some internal shear) in the manual version (Fig. 1).

Reconstructions of the complete pattern of stripes are illustrated in Figure 6. The upper version (Fig. 6A) is from the computer model, the lower version (Fig. $6 B$ ) is from the manual reconstruction. In both cases, the reconstruction is complete except for a very small region where tissue was damaged during division of the occipital lobe into two blocks.

Comparing the two versions (which were prepared completely independently), it is clear that they differ considerably from each other in the details of the pattern of stripes. Some of these 


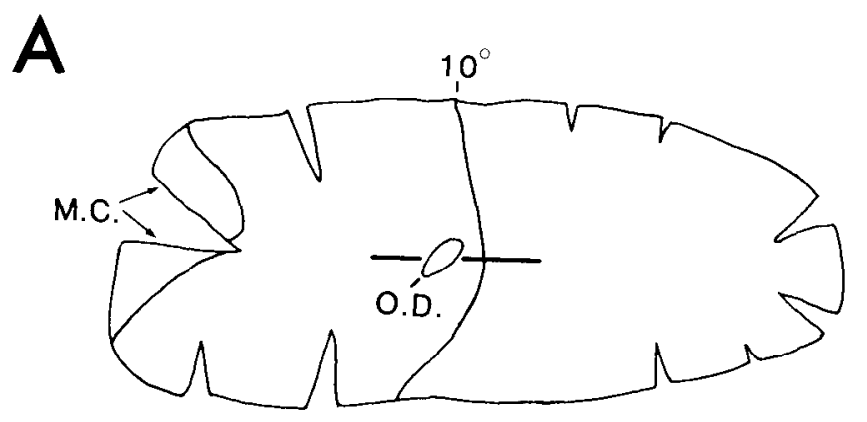

B

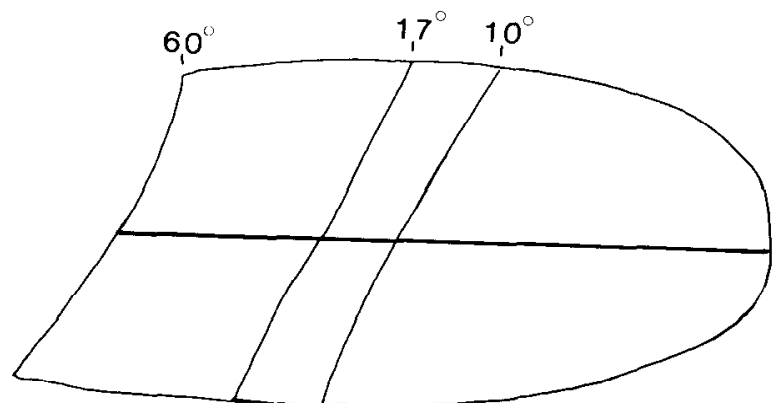

Figure 3. Matching of cortical maps from the computer reconstruction $(A)$ and from the mathematically generated model $(B)$. The computer reconstruction shows the location of known topographic coordinates, including the $10^{\circ}$ isoeccentricity contour, part of the horizontal meridian, the optic disc $(O . D$.$) representation \left(17^{\circ}\right.$ eccentricity), and the monocular crescent (M. C.; approximately $60^{\circ}$ eccentricity). The mathematical model includes the same set of topographic coordinates; these are approximately in agreement with the actual cortical reconstruction.

differences are distortional (non-topological) in nature. In regions where the cortex is nearly flat, the computer version is probably less distorted than the manual version. This is because the manual version, while allowing minimal distortion of surface area (see "Materials and Methods"), does have significant angular distortions that result in shearing of the stripe pattern in places. However, in regions of high curvature or folding, the manual version is probably more reliable, because the computer version is subject to foreshortening and to possible errors in the joining of segments.

There are also many topological differences between the two reconstructions. These must arise from ambiguities in the labeling that permitted different decisions as to the locations of branch points and islands (see "Materials and Methods"). We have not attempted to resolve these discrepancies by a detailed re-examination of the original micrographs, and their existence clearly limits the reliance that should be placed on the minute details of the stripe pattern. Again, however, we believe that the computer version is more accurate in the open expanses of cortex, and the manual version is more accurate in the highly curved regions.

In spite of these differences of detail, the two versions are in agreement on the general layout and appearance of the columns on the unfolded cortex. There are several features of the pattern that we wish to point out.

1. In agreement with previous work, the stripes form a system of parallel bands, with numerous branches and islands.

2 . The stripes are roughly orthogonal to the V1/V2 border throughout the entire binocular segment of the cortex. This finding extends observations made in opercular cortex with the Liesegang silver method (LeVay et al., 1975).

3 . On the operculum, the stripes initiating from the dorsal and ventral margins of the striate cortex converge and stream medially. In the lateral and central parts of the operculum, where the fovea and the first few degrees of the horizontal meridian are represented, the columns are more disorderly and fragmented than elsewhere.

4. In the periphery the stripes are arranged in a comparatively simple fashion: they run directly across the map, from the dorsal to the ventral border of the striate cortex.

5. In the periphery there is a marked imbalance between the width of the columns for the two eyes. Starting at the lateral margin of the representation of the optic disc (i.e., at an eccentricity of about $20^{\circ}$ ), the columns for the ipsilateral eye (the labeled bands in the reconstruction) become progressively narrower. By the time one reaches the edge of the monocular segment, they have fragmented into strings of small islands.

6 . The average periodicity, based on 200 arbitrarily chosen sites on the manual reconstruction, was $0.88 \mathrm{~mm} /$ pair $( \pm 0.028$, $\mathrm{SEM})$. There is a change in the overall periodicity of the stripes as one moves from the representation of central vision to the far periphery. On the manual version the perimeter of the entire V1/V2 border (except the monocular crescent) is 124 $\mathrm{mm}$, along which there are 118 pairs of stripes, for an average spacing of $1.05 \mathrm{~mm} /$ pair. (For the computer version there are 113 pairs in $107 \mathrm{~mm}$.) Considering only the perimeter of the operculum, the average width of a left-plus right-eye pair is $1.14 \mathrm{~mm}$. In the cortex, just ventral to the monocular segment (representing the superior periphery), a pair of columns averages $0.64 \mathrm{~mm}$ in width. Thus, ocular dominance "hypercolumns" (Hubel and Wiesel, 1974) are almost twice as wide centrally as in parts of the far periphery. The reduction in their size in the periphery is not accounted for entirely by the reduction in width of the ipsilateral eye's columns: the contralateral eye columns also become narrower, although to a lesser extent.

7. There are also local fluctuations in the periodicity of stripes that are not obviously related to visual topography. The two reconstructions differ considerably in this respect: the manual version (Fig. $6 B$ ) shows more local variability than does the computer montage (Fig. 6A). The computer version may underestimate the variability in some regions owing to incomplete correction of foreshortening, especially along the margins and in regions of high curvature. The manual version may include some artifactually narrow or wide stripes as a result of incorrect matches or shearing during the unfolding process. We took some regions that appeared to be accurately reconstructed in the manual version and re-examined the stripe width in the original photographs. From these measurements and from the photomontage of the opposite hemisphere (see below and Fig. 7), we concluded that local variability (within a distance of about 10 stripes' width) was generally quite modest, with fluctuations of about $50 \%$ being common. Not infrequently, however, 2-fold fluctuations were encountered, and there were occasional but convincing examples of 3 -fold differences between nearby stripes. Some of this variability may be related to the constriction of stripes at branch points (Swindale, 1980). There may also be a tendency for more pronounced variability in regions where the cortex is folded. These local fluctuations, when coupled with the systematic regional variations noted above, lead to overall extremes of periodicity which span a 4-fold range: 2.1 versus $0.5 \mathrm{~mm} /$ pair averaged over two pairs of stripes in regions of particularly narrow spacing (ventral to the monocular crescent) and wide spacing (midway along the inferior vertical meridian representation).

8. The representation of the optic disc occupies an elongated 

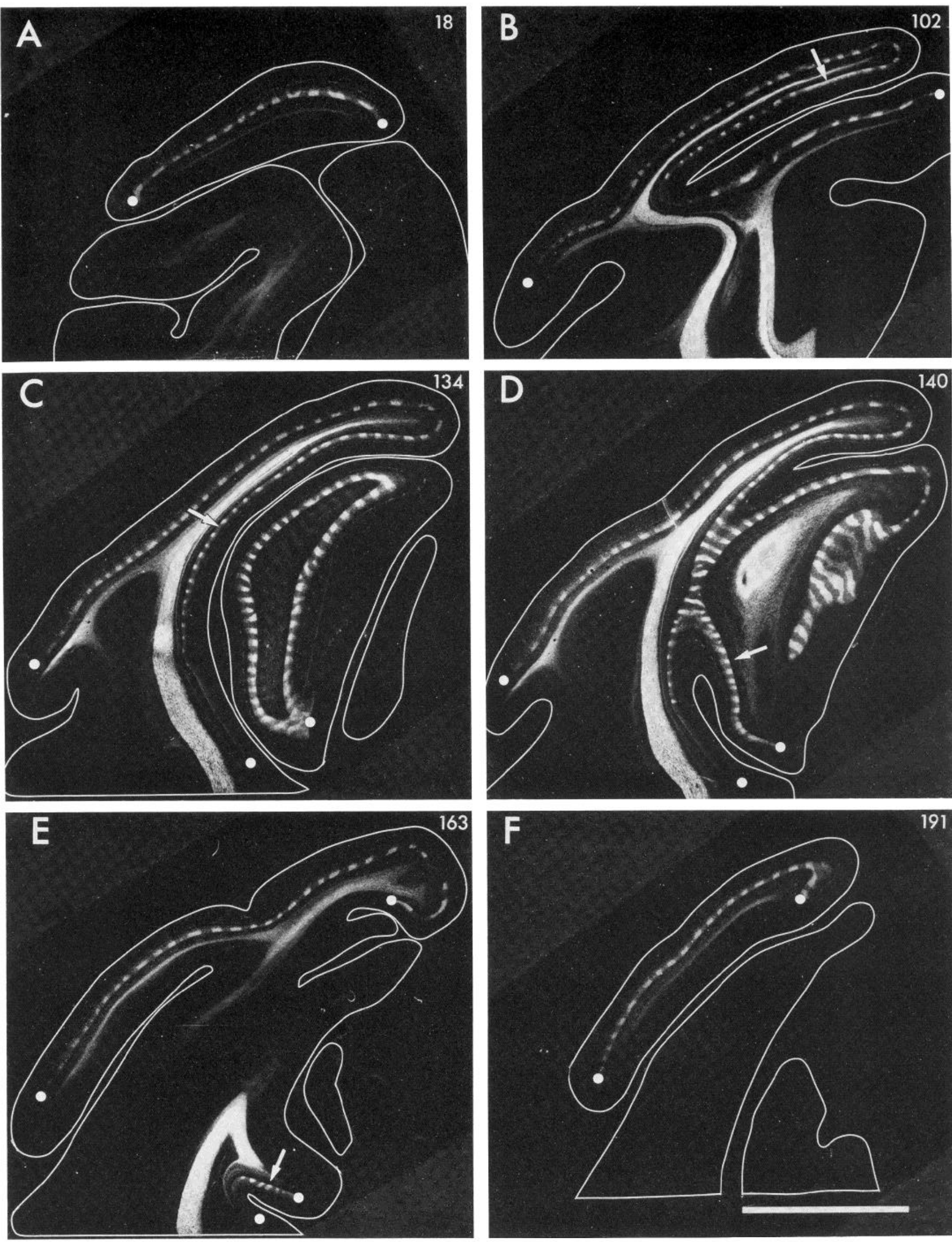

F

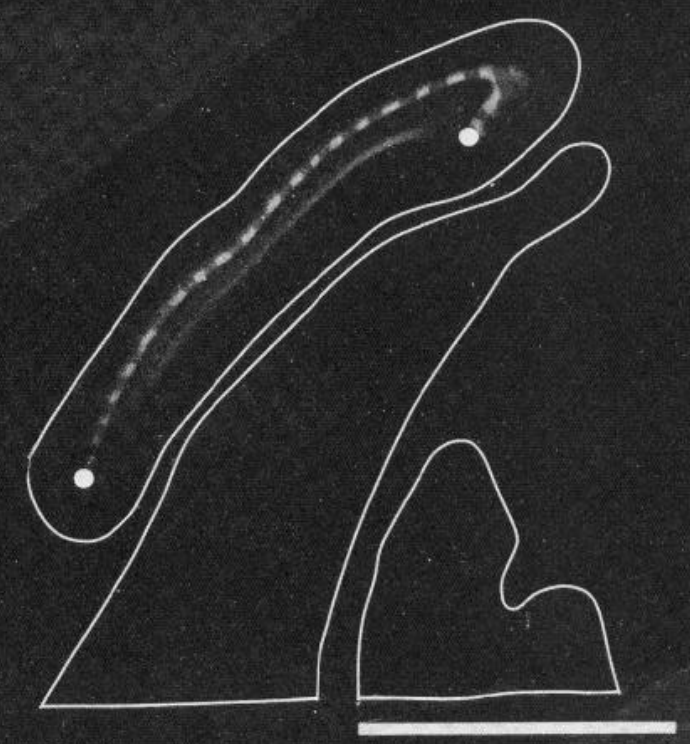



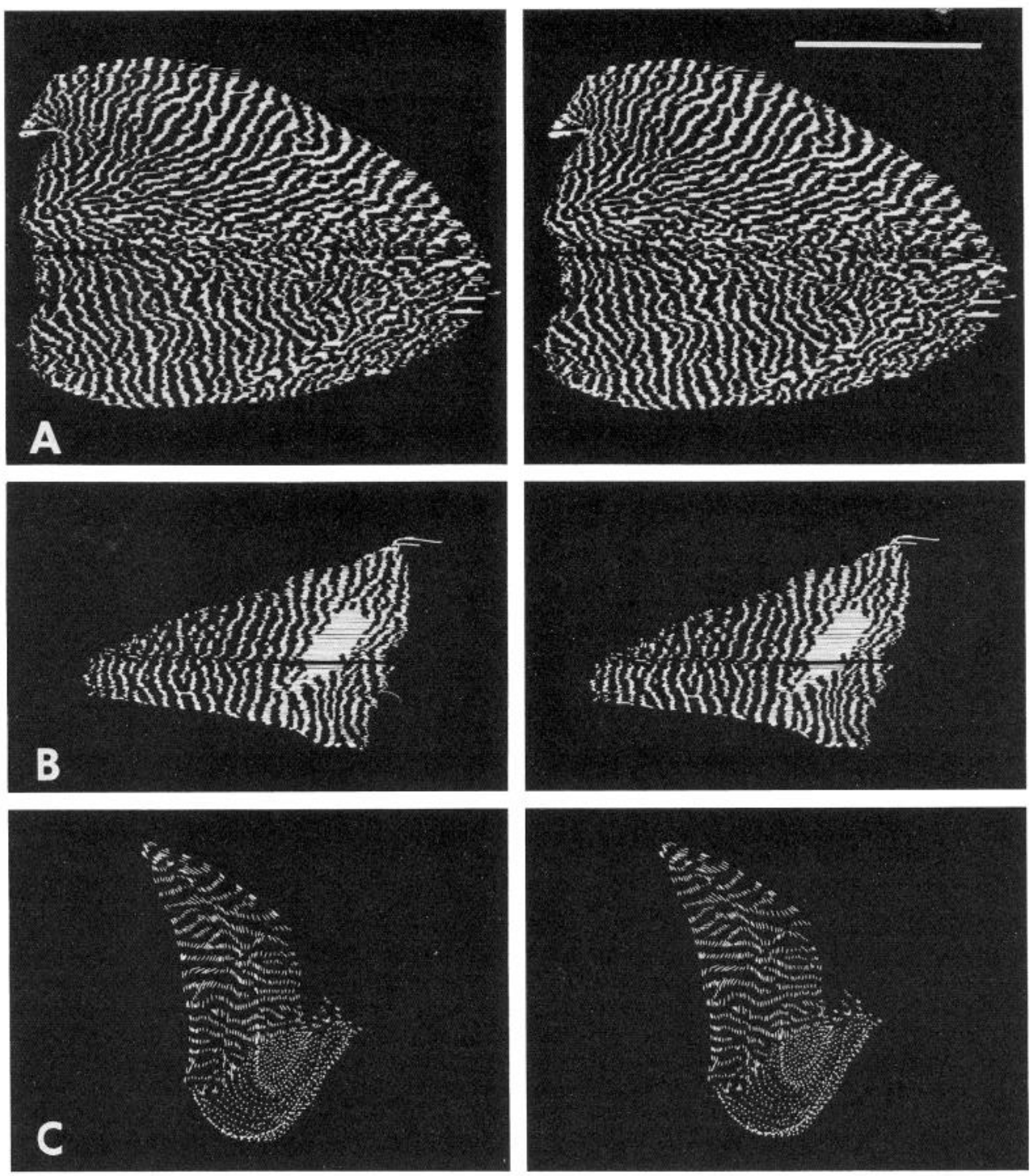

Figure 5. Stereo pairs of reconstructed ocular dominance stripes for three (from a total of eight) segments of striate cortex. The images were photographed directly from the computer screen, with a $6^{\circ}$ angle between views. Each segment is viewed en face and with its pial surface toward the viewer. The scale bar is $1 \mathrm{~cm}$. A, Opercular segment (medial is to the left; dorsal is up: same orientation as in the final reconstruction of Fig. $6 \mathrm{~A}$ ). The surface is gently domed with a shallow V-shaped depression pointing laterally. The perimeter of the segment, except for the medial edge, forms the V1/V2 border. This is one of five views of this segment that were used in making the final reconstruction. $B$, Roof of the calcarine fissure (medial, to the right; dorsal, up: same orientation as in the final reconstruction). Medially, this segment abuts the opercular segment. None of the perimeter of this segment forms the V1/V2 border. The elongated, solidly labeled patch is the representation of the contralateral eye's optic disc. Lateral to the disc, the labeled stripes become narrower than the gaps between them. This segment is relatively flat, except near its edges. The unlabeled gap running horizontally across the entire segment is the "seam" between the two tissue blocks; due to an error in data entry, it is represented at about twice its proper width. $C$, Dorsal bank of the calcarine fissure (medial is to the left; anterior is down: the segment is rotated about $90^{\circ}$ counterclockwise with respect to its orientation in the final reconstruction). The left-hand edge of the segment forms the V1/V2 border. The segment has a vertically elongated depression toward its right-hand side (a ripple in the stem of the calcarine fissure). The seam between the block slices through this depression and makes it appear somewhat too deep. The monocular segment (dotted region) lies in the sharply curved base of the fissure. area, about $7 \times 3.5 \mathrm{~mm}$ in size, in the roof of the calcarine fissure. The position of the disc in the retina is known to be on the horizontal meridian $17^{\circ}$ from the fovea (Malpeli and Baker, 1975), which provides an additional landmark for aligning the columnar reconstruction to the map of the visual field. The disc itself is slightly elongated $\left(7.2^{\circ} \times 5.3^{\circ}\right.$, according to Malpeli and Baker, 1975) with long axis vertical. The tilt of the long axis in the cortex with respect to the horizontal meridian representation may reflect the oblique orientation of isoeccentricity lines in parts of the periphery of the field map (see below and Fig. $3 A$ ). The ocular dominance stripes in the neighborhood of the disc are not oriented in any special fashion with respect to it, but merely flow past it.
The right hemisphere of the same brain (contralateral to the injected eye) was sectioned tangential to the center of the operculum and the roof of the calcarine fissure (see "Materials and Methods"). Autoradiographic montages were prepared from these two regions and are illustrated in Figure 7 . On the operculum (Fig. 7A) the columns form the familiar pattern, streaming away from the V1/V2 border and medially toward the lip of the calcarine fissure. The labeled and unlabeled bands are about equally wide throughout this region. In the roof of the fissure (Fig. $7 B$ ) the optic disc is represented by an unlabeled patch that is once again elongated in shape. Just as was seen in the left hemisphere, in the region lateral to the disc,

Figure 4. Six examples $(A$ to $F$ ) from the complete set of 203 autoradiographs used in the reconstruction. They are horizontal sections through the occipital lobe ipsilateral to the injected eye. Posterior is up, medial is to the right. The scale bar is $1 \mathrm{~cm}$. White dots mark the position of the border of striate cortex wherever it appears in the sections. These border crossings have marked on the cortical map in Figure $1 B$. The dorsalmost and ventralmost sections $(A$ and $F)$ pass through the opercular portion of striate cortex only; the intervening sections include both the opercular region and portions of the calcarine fissure. The optic disc of the contralateral eye is represented by a small zone of continuous heavy labeling in the roof of the calcarine fissure (arrow in $B$ ). the monocular crescent is represented by a larger region of continuous, weak labeling on the dorsal bank of the calcarine fissure (bottom center in $C, D$, and $E$ ). Note the regional variations in the appearance of the ocular dominance patches, especially the imbalance between the widths of the labeled and unlabeled patches in the region between optic disc and monocular segment (e.g., at the arrow in $C$ ), and the close spacing of patches in parts of the calcarine fissure (e.g., arrows in $D$ and $E$ ) compared with the spacing on the operculum. 


\section{A}
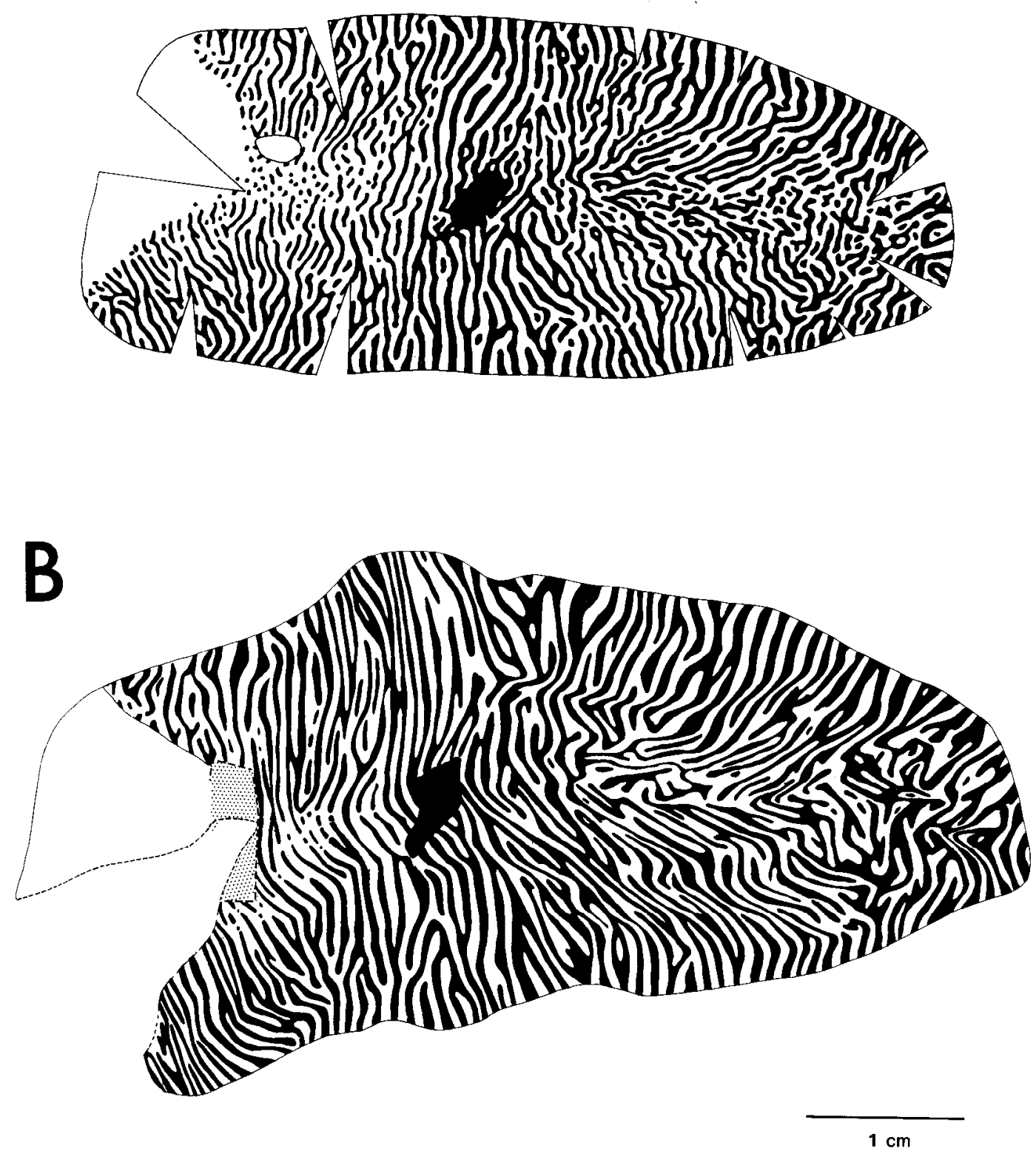

Figure 6. The complete pattcrn of stripes in the computer reconstruction $(A)$ and the manual reconstruction $(B)$. The orientation of the reconstructions is the same as in Figure 1, with the operculum to the right and dorsal upward.

the stripes for the contralateral eye (in this case the labeled stripes) are wider than those for the ipsilateral eye.

Since this narrowing of the ipsilateral eye's stripes was seen on both sides of the brain, it seemed unlikely to be an artifact due, for example, to damage to the injected eye. Even so, given that this effect had not been described previously, we thought it possible that it was a peculiarity of this particular monkey. We therefore examined the brains of two other monkeys that had received eye injections. They both showed the same imbalance in the periphery. Montages of the roof of the calcarine fissure are illustrated for one of these monkeys in Figure 8. It appears, therefore, that the region of cortex carrying the representation of the portion of the visual field peripheral to the optic disc is dominated by input from the contralateral eye. It is also apparent from both of these montages that the optic disc representation is distinctly elongated, although the recon- structions are not complete enough to determine their precise dimensions.

Transformation of stripes onto the visual field. Using recent data on the topographic organization of striate cortex (Van Essen et al., 1984), in conjunction with limited mapping data from the experimental hemisphere used in this study, we calculated the complete pattern of stripes, as they would appear when transposed back to the visual field (see "Materials and Methods"). Figure 9 shows the pattern for the entire pattern out to $60^{\circ}$ eccentricity, which is close to the monocular cresent (lower inset; see Fig. 9 legend). The pronounced curvature of the vertical meridian along the right margin of the map is, as was described under "Materials and Methods," an intentional consequence of the procedure used to construct a flattened representation of the visual hemifield.

There are several interesting features of these illustrations that deserve specific mention. Most obvious is the enormous 

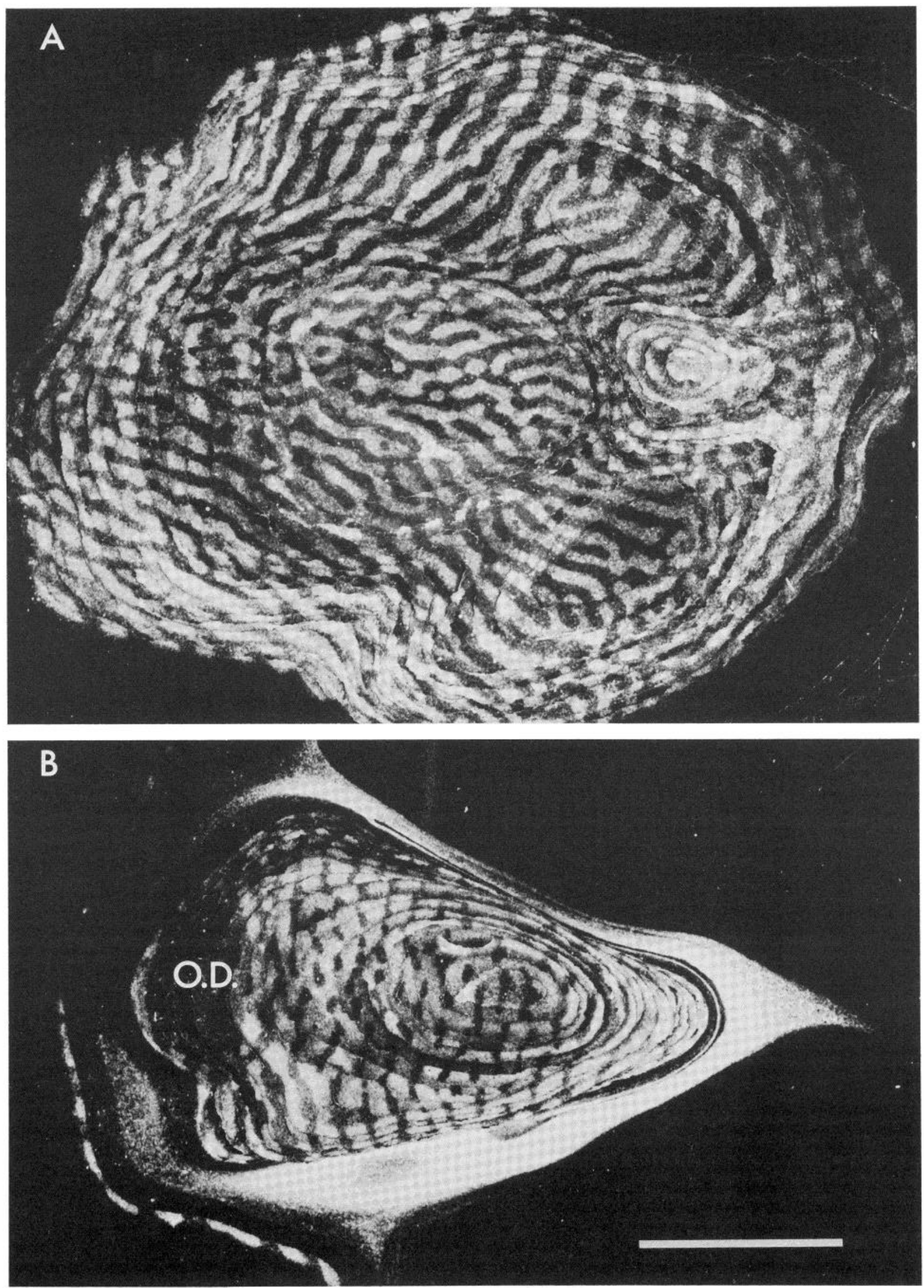

Figure 7. Photographic montages of the ocular dominance stripes in the hemisphere contralateral to the injected eye, prepared from tangential sections. A, Opercular segment (medial is to the left, dorsal is up). The reconstruction covers only about $60 \%$ of the opercular surface and nowhere reaches the V1/V2 border. Some idea of the curvature of the surface can be gained by inspection of the "contour lines"-autoradiographs were photographed at $100-\mu \mathrm{m}$ intervals. Compare with Figure $5 A$. B. Roof of the calcarine fissure (same orientation as $A$ ). The reconstruction does not extend to the medial lip of the fissure. The injected eye's optic disc is represented by an unlabeled zone medially $(O . D$.). Lateral to the disc the labeled stripes (those for the contralateral eye) are wider than the gaps between them. Contrast this with the pattern of narrow labeled stripes in this region of the opposite hemisphere (Fig. $5 B$ ) and with the equally wide stripes and gaps on the operculum. Scale bar $=5 \mathrm{~mm}$. 


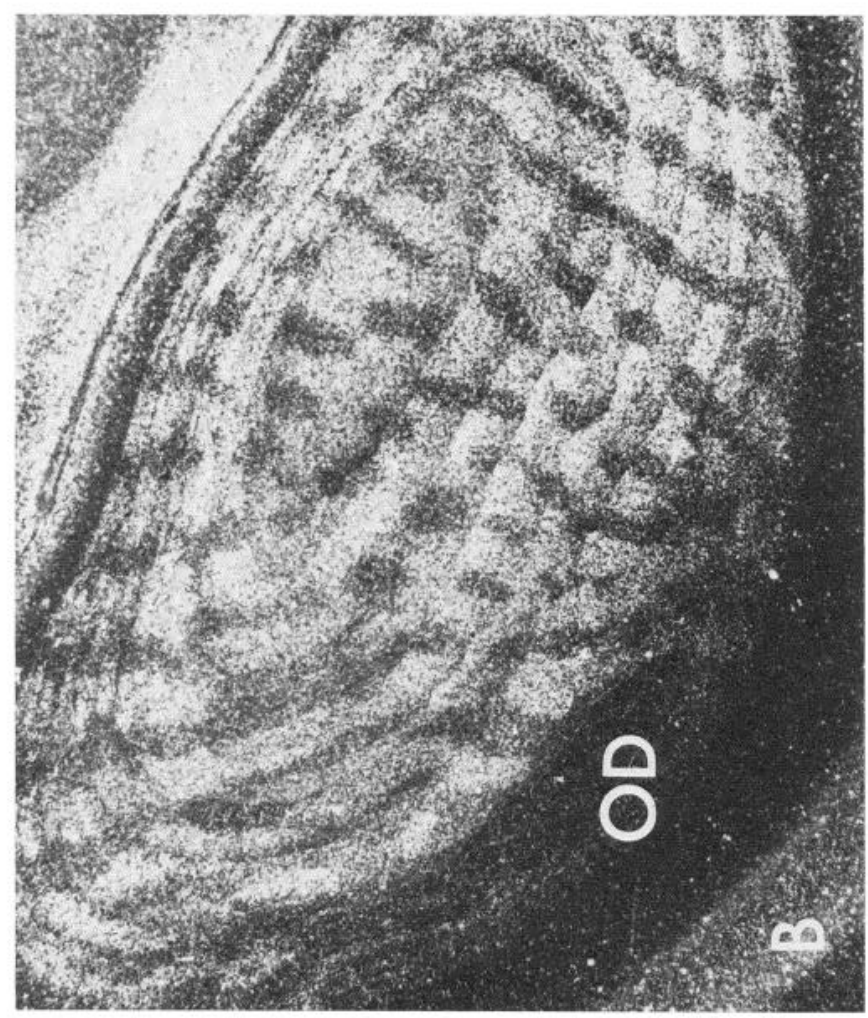

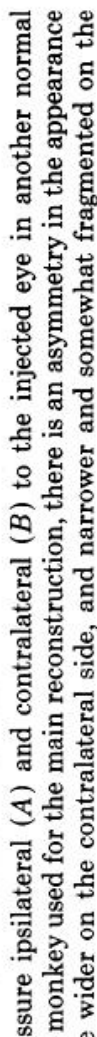

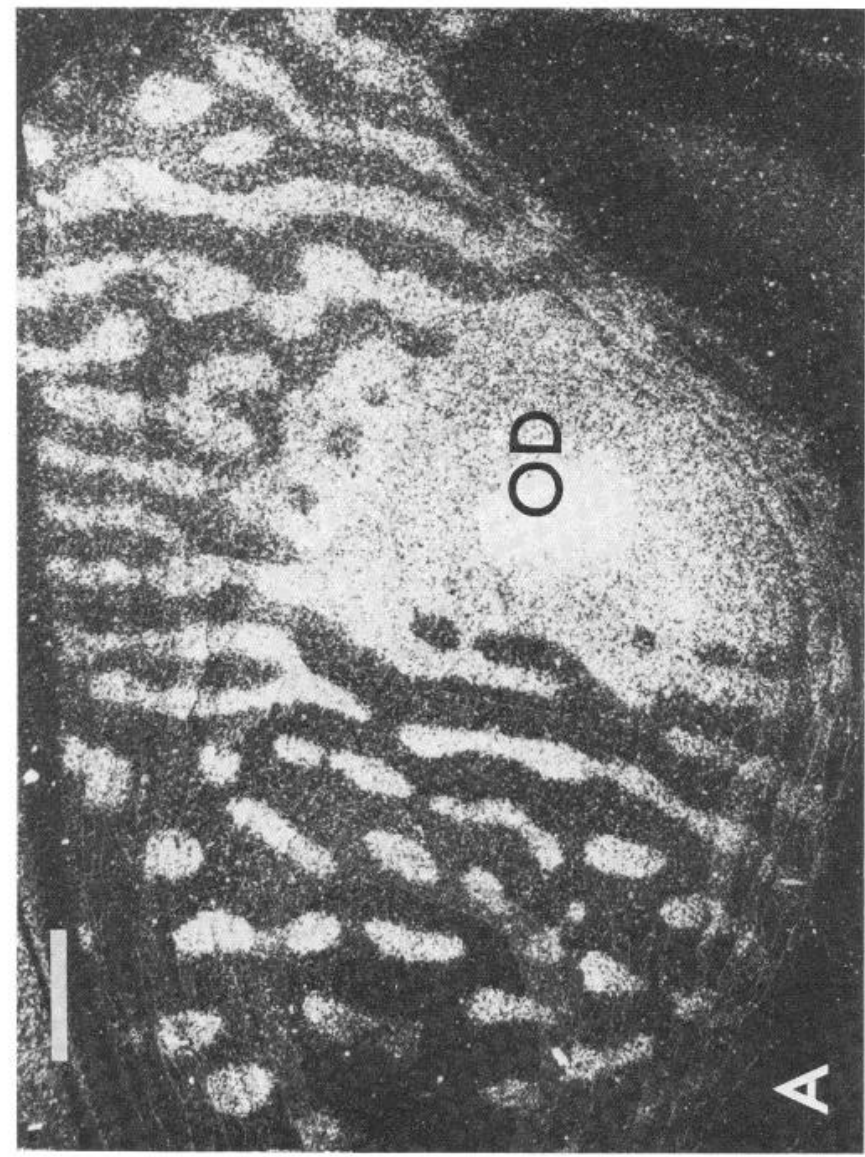

造

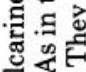

ฮั

ฐ

녕 뎽용

岁. 현

옥 웜

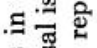

造

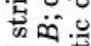

ㄷ.

हี .

这

용

प혀

吨.

녀예

.

웅요

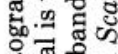

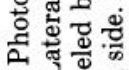

$\infty$ 造

s. 


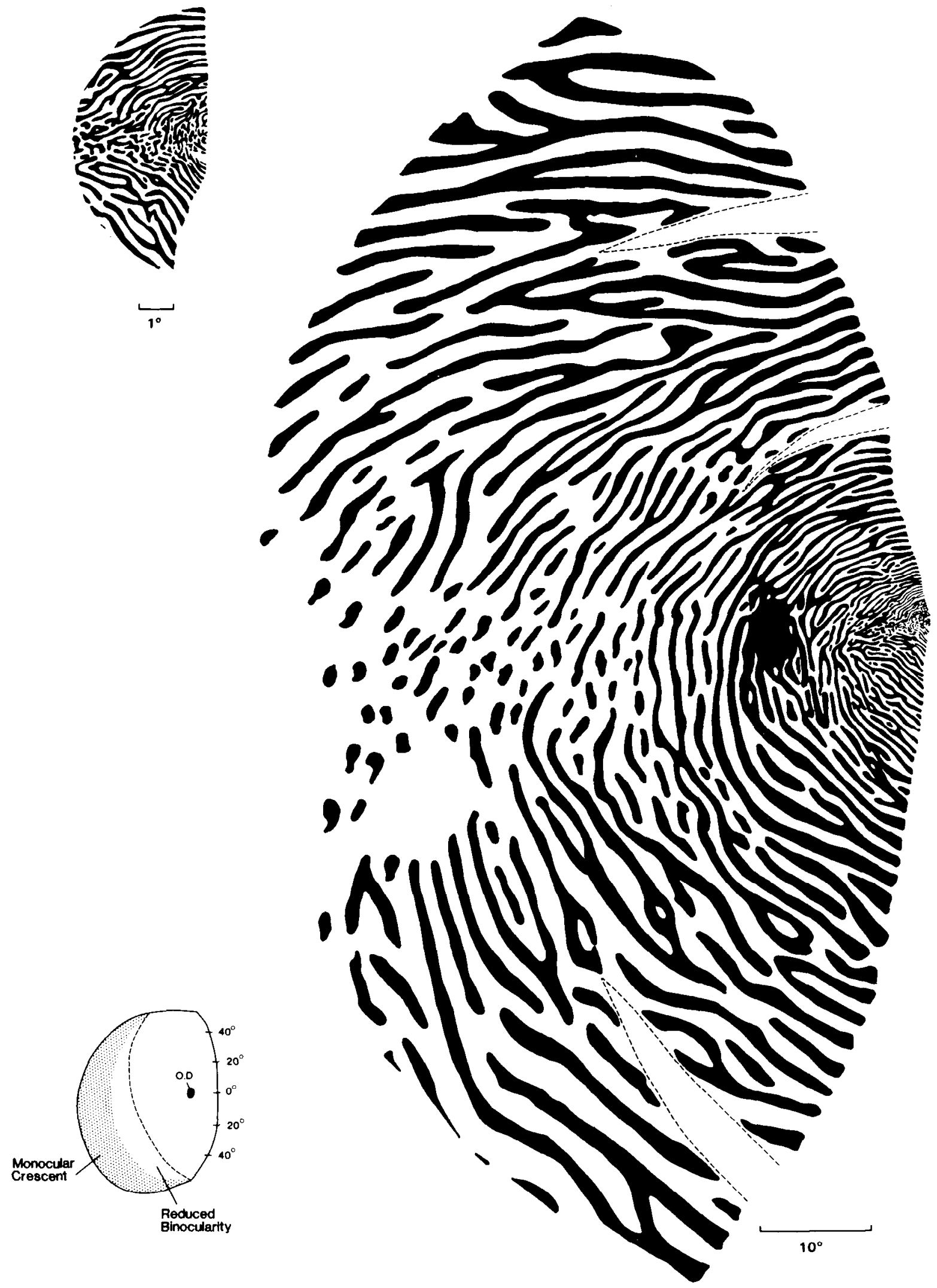

Figure 9. The complete pattern of ocular dominance stripes back-transformed onto the visual field out to $60^{\circ}$ eccentricity. The optic disc appears more elongated than in the actual retina because no correction was made for the local anisotropy involved in its cortical representation (see "Discussion"). The small oval blank region in the far periphery, just below the horizontal meridian, corresponds to the artifactual gap in the cortical reconstruction (Fig. 6A; see "Materials and Methods"). The inset in the upper left shows the transformation for the central $4.5^{\circ}$. The lower left inset shows the extent of the entire left visual hemifield, from Polyak (1957). The dashed line shows the extent of the right eye's field of view when the direction of gaze is straight ahead. The stippled region shows the approximate extent of the true monocular crescent, in which the retinal extent of the left eye exceeds that of the right eye. This was based on the perimetry measurements on one of us (D. V. E.) with different directions of gaze for the right eye. 
difference in spacing of stripes between the fovea and the visual periphery. In the center of the fovea (Fig. 9, upper inset) there are about 8 pairs of stripes/degree, whereas in the far periphery, each pair of stripes occupies several degrees. Outside $5^{\circ}$ to $10^{n}$, the stripes show a strong tendency to stream in parallel arcs that spiral inward while progressing from the inferior to the superior vertical meridian. The fact that their course is not exactly parallel to isoeccentricity contours is a reflection of the oblique orientation of isoeccentricity contours in regions where the stripes run approximately vertically on the map. The actual misalignment is even greater than it appears in this illustration, however, because our computer model did not fully reflect the asymmetric orientation of isoeccentricity lines in the periphery (see Fig. 3 and "Materials and Methods"). A completely accurate back-transformation would, for example, show a tendency for stripes starting at $20^{\circ}$ eccentricity to spiral inward to less than $10^{\circ}$ at the superior vertical meridian.

\section{Discussion}

This study represents the first successful reconstruction of the complete system of ocular dominance stripes in primate striate cortex. Our results have in large part confirmed a number of previous observations about the stripes, but several new and unexpected observations have arisen as well.

By using two entirely independent methods of cortical reconstruction, we have been able to assess the overall validity and the relative strengths and weaknesses of the different approaches. The maps are quite similar in overall shape and in the location of major landmarks, although there are some regions where the differences are significant. There are also many topological differences in terms of the detailed pattern of stripes throughout the map. This was unexpected when we first compared the two maps, but in retrospect it is perhaps not so surprising. The overall pattern was determined from 200 sections, most containing between 30 and 80 labeled stripes, for an estimated total of more than 10,000 matches between adjacent sections. Thus, even if $99 \%$ of the match-ups between sections were correct on each map, there would nonetheless be hundreds of discrepancies between maps.

Throughout the striate cortex, the ocular dominance columns occur as alternating stripes, but there are significant regional variations in their dimensions and orientation. Some of these variations appear to be largely random, but others are systematically related to the location in the cortex. Thus, the average periodicity is significantly less in the far periphery than more centrally, but this is superimposed on local fluctuations in periodicity that are up to severalfold in magnitude. A related finding by Livingstone and Hubel (1984) is that the density of cytochrome oxidase patches increases moderately with eccentricity, from $4.5 / \mathrm{mm}^{2}$ at $0^{\circ}$ to $6.5 / \mathrm{mm}^{2}$ at $8^{\circ}$.

We have confirmed previous reports (Kennedy et al., 1975; LeVay et al., 1980) that the optic disc representation in striate cortex is located in the calcarine sulcus, in the smooth region directly under the operculum. Its exact location is variable, though, which presumably correlates with individual variability in topographic organization (Van Essen et al., 1984). The ocular dominance stripes which adjoin the optic disc representation do not intersect it at a specific angle; rather, their course seems largely oblivious of its presence. Thus, it does not appear to be an organizing center for stripe formation, at least in the monkey.

An intriguing feature of the optic disc representation is its elongated shape, which is suggestive of a local anisotropy in the cortical magnification factor. The cortical representation of the optic is about twice as long as it is wide, whereas the disc itself is only slightly elongated. It measures about $7^{\circ} \times 5^{\circ}$ according to Malpeli and Baker (1975), and in the particular monkey used in our study the discs appeared very close to circular on ophthalmoscopic examination. One clue as to the basis of this anisotropy is that Hubel et al. (1974b) reported an anisotropy in the representation within layer $4 \mathrm{C}$, with the magnification factor parallel to ocular dominance stripes being approximately twice as large as that perpendicular to the stripes. The optic disc can thus be regarded as an enlarged region of strictly monocular input, but with basically the same type of anisotropic organization.

Another clue is that the representation of the optic disc in the LGN is also quite elongated, even more than in the cortex, and the axis of elongation is roughly parallel to isoeccentricity contours (Connolly and Van Essen, 1984). The greater degree of elongation in the LGN is probably related to an encroachment from surrounding parts of the representation and a net reduction in size of the optic disc gap relative to the extent expected if projection lines were completely free of distortion in that region (Malpeli and Baker, 1975). In striate cortex the expected size of the optic disc representation about $9 \mathrm{~mm}^{2}$, based on the magnification factor in this region $\left(0.3 \mathrm{~mm}^{2} / \mathrm{deg}^{2}\right.$, from equation 2) and the size of the optic disc (30 $\left.\mathrm{deg}^{2}\right)$, which is an ellipse of $7.2 \times 5.3^{\circ}$ (Malpeli and Baker, 1975). This is in good agreement with the measured area of $8.8 \mathrm{~mm}^{2}$ for the computer map (upon which equation 2 was based) and $14 \mathrm{~mm}^{2}$ for the manual map. Thus, the cortical representation of the optic disc does not appear to be grossly perturbed by interactions between the two eyes.

The monocular crescent provides another region in which interesting comparisons can be made of the retinal, geniculate, and cortical representations. In the visual field, the monocular crescent is a large, somewhat asymmetric zone occupying about $25 \%$ of the hemifield and with a maximum width about onefifth of its length (Fig. 9, lower inset). In the unfolded maps of the striate cortex, the monocular crescent is, as expected, much smaller in relative extent ( 7 to $8 \%$ of total surface area). It is also much less elongated, having approximately the shape of an equilateral triangle. This is suggestive of an anisotropy involving compression along isoeccentricity contours, rather than the elongation found for the optic disc. In contrast, the monocular crescent representation in the LGN, as identified by the region in which ipsilateral and contralateral eye layers do not overlap, is highly elongated, even more so than in the visual field. However, the extent of this non-overlap region is only $4 \%$ of total laminar surface area, despite the fact that the overall emphasis on the visual periphery is greater in the LGN than in striate cortex (Connolly and Van Essen, 1984). This suggests that lines of projection may be perturbed in this region, as alluded to already for the optic disc, and that the true monocular crescent in the LGN may be significantly wider. Hence, the relationship between LGN and cortical anisotropy in this region remains unclear.

Ocular imbalance. The normal imbalance between ipsilateral and contralateral eye inputs in the visual periphery was not explicitly mentioned in previous studies of ocular dominance stripes, presumably because it is particularly obvious, mainly in the irregularly folded cortex in the anterior calcarine sulcus. It appears to have been noticed but confused with a deprivation effect by Swindale et al. (1981). ${ }^{4}$

There are two obvious possibilities for explaining the imbalance of inputs at the cortical level. The first is that the imbalance might reflect a naturally occurring form of visual deprivation, given that the anatomical pattern closely resembles that seen after experimentally produced monocular deprivation

\footnotetext{
${ }^{4}$ The autoradiographic observations on visually deprived monkeys by Hubel et al. (1977) and LeVay et al. (1980) were restricted almost entirely to opercular cortex, and the interpretation of those observations is not affected by the present findings.
} 
(Hubel et al., 1977; LeVay et al., 1980). The basis of this deprivation would be the obstruction of the field of view by the nose. Because of eye movements, the degree of deprivation would vary continuously over an extended eccentricity range centered at about $45^{\circ}$ eccentricity, which is where the obstruction begins when the eyes are directed straight ahead. The second possibility is that the imbalance reflects the known anatomical asymmetry in retinal ganglion cell density between temporal and nasal retina (Van Buren, 1963; Stone and Johnston, 1981). From Van Buren's (1963) data on the human retina (Fig. 36 of Van Buren, 1963), cell densities are symmetrical for eccentricities less than $15^{\circ}$, but outside of this range, the nasal (contralaterally projecting) retina has approximately twice the cell density of the temporal (ipsilaterally projecting) retina The retina imbalance is thus present in the appropriate region and is quantitatively sufficient to account for the imbalance seen at the cortical level. If this asymmetry is indeed a contributing factor, it would imply that differences in the number of inputs have the same effect as differences in activity levels in establishing the relative extent of target areas between competing inputs, as has been suggested previously for the cat (LeVay et al., 1978).

Transposition to the visual field. Hubel and Freeman (1977) used information from an earlier study (Iubel and Wiesel, 1974) for estimating the cortical magnification factor as a function of eccentricity. Their equation 1 was an expression for the inverse of the linear magnification factor

$$
M^{-1}=0.0637 E+0.109
$$

This can be rearranged to give an explicit equation for the linear magnification factor, $M_{\mathrm{lin} 1}$

$$
M_{\text {lut }}=15.7(E+1.62)^{-1}
$$

Since they assumed that the representation was uniformly isotropic (outside layer $4 \mathrm{C}$ ), this expression can be squared to give their estimate of the areal magnification factor, $M_{\text {o }}$

$$
M_{a}=246(E+1.62)^{-2}
$$

The exponent of this power function determines how steeply the magnification factor declines with eccentricity outside the foveal region. A value of precisely -1 for linear magnification is mathematically very convenient, because it makes it possible to integrate both sides of the equation and hence to obtain explicit expression for distance in the cortex as a function of eccentricity. This is the so-called "logarithmic conformal" mapping described by Fischer (1973) and Schwartz (1980).

In the present study we obtained an expression for the areal magnification factor (equation 2) which had the same exponent as estimated by Hubel and Freeman (1977) but somewhat lower values for the other two constants in the equation. In particular, our estimate was 100 versus 246 for the multiplicative constant, $a$, which determines the overall size of the map, and 0.82 versus 1.62 for the additive constant $b$, which determines the eccentricity range over which the magnification factor reaches a plateau for the fovea, rather than increasing without limit. These differences are not enormous, but they do affect several quantitative conclusions. For example, application of our computerized modeling algorithm to the Hubel and Freeman (1977) expression for magnification leads to a model of striate cortex with a surface area of $2120 \mathrm{~mm}^{2}$, which is substantially larger than any actual striate cortex of $M$. fascicularis examined in the study of Van Essen et al. (1984) (range, 690 to $1560 \mathrm{~mm}^{2}$, $n=31$; Van Fssen et al., 1984). This presumably accounts for their slightly higher estimate of the number of stripes encountered in a traverse of the full eccentricity range (75 left-right pairs versus our direct counting of slightly fewer than 60 ).

In a formal sense, the information content of the pattern of back-transformed stripes is entirely derived from. and thus contained within, the cortical maps of stripes (Fig. 6) and visual topography (Fig. 3). Nonetheless, the transformation provides a particularly clear illustration of several major points about the cortical representation of the visual field. Most obvious is the roughly 40 -fold range in the width of stripes between the fovea and the far periphery. Of course, this is predictable from the knowledge that the cortical spacing between stripes decreases by about a factor of 2 with eccentricity, whereas the linear magnification factor, based on equation "2, changes 76 . fold between $0^{\circ}$ and $60^{\circ}$. The visual impression of the difference between center and periphery is even larger, though, because the density of stripes of a given length is inversely proportional to areal, not linear magnification, which spans a $5800-\mathrm{f}^{\prime} \mathrm{ll}$ range between extremes of the center and periphery.

Another feature of the back-transformation suggests a pos sible psychophysical correlate of cortical anatomy. This is the ocular imbalance favoring contralateral over ipsilateral inputs in the visual periphery. The imbalance is evident only in a small portion of the cortical map but in a much larger fraction of the visual field along the horizontal meridian and in the lower quadrant. M. Fahle and M. Schmid (personal communication) have measured monocular hyperaruity for stationary and moving verniers at different positions along the hurizontal meridian in human subjects. They find that it is similar in nasal and temporal hemifields out to the optic disc. but beyond the disc it falls off much more steeply in the nasal than in the temporal hemifield. The correspondence bet ween these psychophysical observations and our present anatomical results is consistent with the notion that hyperacuity is limited by the magnification of the retinal image in laver 4 (") the cortex (Barlow, 1981; Crick et al., 1981).

One might also expect to find a psychophysical correlate of the inward spiraling of back transformed stripes as they progress toward the superior vertical meridian. Presumably, this would be manifested by a bias favoring upper fields at cent ral eccentricities (up to $10^{\circ}$ or $20^{\circ}$ ) and a reversed bias, favoring lower fields, in the periphery. However. any such bias would be superimposed upon two other asymmetries: st riate cortex usually has more surface area devoted to the lower than to the upper quadrant and more area to the region of the horizontal meridian than to the vertical meridian (Van Fisen et al., 1984) There is substantial individual variabilitv in all of these cortical asymmetries, though, and any analysis of psychophysical asym metries should take such variability into account.

Developmental significance. When large-scale reconst ructions of ocular dominance stripes were first obtained (LeVay et al., 1975), it was suggested that the stripes arose because of a conflict during development between two opposing instructions. One of these was for both left-and right-eve afferents to occupy the same cortical area according to a single retinotopic map, and the other was a force tending to keep the two sets of afferents separate from each other. The segregating influence was suggested to be a repulsive force between afferents from different eyes, although it could equally well be an attractive force between afferents from the same eye. Alternating bands arise in this situation because they minimize the interface between the two sets for a given degree of interpenetration (as compared with other possible configurations such as islands in a matrix). The periodicity of the bands is set by the relative strengths of the two forces. Attempts to model st ripe development on the basis of these or related ideas (von der Malsburg, 1979; Swindale, 1980; Fraser, 1984) have shown that, although stripes are indeed generated, they show only local order. For the stripes to remain parallel over any considerable extent of cortex requires the presence of an external, anisot ropic ordering influence. This anisotropy has been suggested to be an elongation of all the geniculocortical aborizations in one direction 
(von der Malsburg, 1979), a gradual anisotropic stretching of the cortex during stripe formation (Swindale, 1980), and/or a difference in the strength of two orthogonal gradients of adhesiveness (von der Malsburg, 1979; Fraser, 1984).

These various descriptions for an anisotropic influence are not mutually exclusive, and they need not represent fundamentally independent mechanisms. We suggest that this issue can best be analyzed by first considering a more basic anisotropy, relating to the boundary conditions for the geniculocortical projection. The largest of the LGN layers are roughly circular in outline (Connolly and Van Essen, 1984), whereas the cortex is approximately twice as long as it is wide, with the long axis oriented horizontally in our standard format. Thus, to a first approximation, it is reasonable to regard the geniculocortical projection as a problem of mapping two circular discs, one for each eye, onto a horizontally elongated ellipse. From a global standpoint, the simplest way of achieving this is to slice each disc into thin vertical strips and then to interdigitate the left cyc and right eye strips, thereby forming an appropriately elongated ellipse. If, instead, one started with discs cut into horizontal strips that were stacked vertically, the strips would have to be stretched horizontally or shrunk vertically by 4 -fold in order to obtain the requisite elongation. More generally, the only way to obtain elongation along one axis without anisotropic stretching of the inputs is to interdigitate strips cut orthogonal to that axis.

'I'his constitutes a general argument for why one might expect stripes to run parallel to the axis of compression of the geniculocortical mapping, which also turns out to be the short axis of the striate cortex. However, the actual determination of stripe orientation during development must be a process involving local interactions of geniculocortical terminals and only indirectly reflecting global constraints. These constraints could he exerted at a local level hy any of several mechanisms including, but not restricted to, those suggested above.

Initially, inputs from the two eyes form separate, completely overlapping projections (Rakic, 1976; Hubel et al., 1977; LeVay et al., 1980), so that the starting condition for ocular segregation is a pair of asymmetrically expanded geniculocortical mappings. Also, it is likely that segregation involves retraction of initially overextended arbors of LGN axons (LeVay and Stryker, 1979). If the global anisotropy is reflected by a corresponding elongation of the terminal arborizations of LGN axons, the process of terminal retraction and segregation would tend to form stripes orthogonal to the axis of elongation (von der Malsburg, 1979).

Why might individual axonal arbors be elongated? One possibility already mentioned is a difference in the strength of two orthogonal gradients involved in establishing topographic organization in the geniculocortical projection (von der Malsburg, 1979; Fraser, 1984). The degree of axonal elongation and also the ease of terminal displacement would be greater along the axis of the weaker gradient, thereby leading to stripes parallel to the stronger gradient. If the two cortical gradients differed by the same absolute amounts at the two extremes, the gradient along the short axis would be roughly twice that along the long axis. Thus, this form of the gradient hypothesis could account for the actual orientation of stripes in a way that is related to the anisotropy in physical dimensions of striate cortex and not to the anisotropy of the geniculocortical transformation. The same hypothesis might account for the less orderly arrangement of stripes near the foveal representation, where the outline of cortex narrows and the gradients might be more isotropic.

A different basis for explaining asymmetries of axonal arborization and subsequent segregation relates to activity patterns and to anisotropies in linear magnification factor in parts of the visual cortex. Several lines of evidence suggest that stripe formation is an activity-dependent process, with the probability of synaptic survival enhanced by synchronous activity (Hubel and Wiesel, 1965; Blasdel and Pettigrew, 1979; Stryker, 1981; Meyer, 1984). There is also evidence for correlated firing patterns of neighboring retinal ganglion cells of like type (Mastronarde, 1983). It is plausible to suppose that the degree of correlation is dependent on retinal ganglion cell separation in a radially symmetric fashion, although this has yet to be experimentally determined. In striate cortex, however, there are several regions where the representation of the visual field is anisotropic. In particular, the representation of the vertical meridian is elongated parallel to the striate border both near the foveal representation, as shown from the ingenious 2 deoxyglucose experiments by Tootell et al. (1982), and in the peripheral representation, as shown by standard electrophysiological mapping (Van Essen et al., 1984). In these regions, correlated activity in a circular region of retina would map to an elongated strip of cortex during the period of binocular overlap. This could contribute to an initial elongation of terminals parallel to the striate border and subsequently to the observed segregation into stripes orthogonal to the border. Along the horizontal meridian, the cortical representation is close to isotropic (Van Essen et al., 1984), and this might be related to the less orderly configuration of stripes in parts of this region.

Finally, what basis might there be for the various regional differences in periodicity of stripes, such as their narrower spacing in the periphery? As noted above, the periodicity may be determined by the relative strength of two forces, one tending to keep opposite-eye afferents apart and the other tending to map them onto retinotopically corresponding sites. There are many ways in which either or both of these forces might vary modestly with eccentricity. One possibility worth specific mention is related to the increased emphasis on central vision in striate cortex relative to that in the LGN (Malpeli and Baker, 1975; Connolly and Van Essen, 1984). This results in more LGN afferents per square millimeter in the periphery relative to the fovea of the striate cortex. Also, the relative input from magnocellular versus parvicellular layers is greater in the periphery than in foveal striate cortex. Such differences in the density or type of afferent fiber might have a significant effect on the range of competitive interactions during stripe formation in the cortex.

\section{References}

Barlow, H. (1981) Critical limiting factors in the design of the eye and visual cortex. Proc. R. Soc. Lond. (Biol.) 212: 1-34.

Blasel, G. G. and J. D. Pettigrew (1979) Degree of interocular synchrony required for maintenance of binocularity in kitten's visual cortex. $\mathrm{J}$ Neurophysiol. 42: 1692-1710.

Connolly, M., and D. C. Van Essen (1984) The representation of the visual field in parvicellular and magnocellular laminae of the lateral geniculate nucleus in the macaque monkey. J. Comp. Neurul. 226 . $544-564$

Crick, F. H. C., D. C. Marr, and T. Poggio (1981) An informationprocessing approach to understanding the visual cortex. In The Organization of Cerebral Cortex, F. O. Schmitt, F. G. Worden, G. Edelman, and S. G. Dennis, eds., pp. 505-533, MIT Press, Cambridge, MA.

Daniel, P. M., and D. Whitteridge (1961) The representation of the visual field of the cerebral cortex in monkeys. J. Physiol. (Lond.) 159; 203-221.

Fischer, B. (1973) Overlap of receptive field centers and representation of the visual field in the cat's optic tract. Vision Res. 13: 2113-2120. Fraser, S. E. (1984) Cell interactions involved in neuronal patterning: An experimental and theoretical approach. In The Molecular Basis of Neural Development, G. Edelman, W. E. Gall, and W. M. Cowan, eds., John Wiley \& Sons, Inc., in press.

Grafstein, B. (1971) Transneuronal transfer of radioactivity in the central nervous system. Science 172: 177-179.

Hubel, D. H., and D. C. Freeman (1977) Projection into the visual field 
of ocular dominance columns in macaque monkey. Rrain Res. 122 : 336-343.

Hubel, D. H., and T. N. Wiesel (1965) Binocular interaction in striate cortex of kittens reared with artificial squint. J. Neurophysiol. 28: 1041-1059.

Hubel, D. H., and T. N. Wiesel (1968) Receptive fields and functional architecture of monkey striate cortex. J. Physiol. (Lond.) 195: 215243.

Hubel, D. H., and T. N. Wiesel (1972) Laminar and columnar distribution of geniculocortical fibers in the macaque monkey. J. Comp. Neurol. 146: 421-450.

Hubel, D. H., and T. N. Wiesel (1974) Uniformity of monkey striate cortex: A parallel relationship between field size, scatter, and magnification factor. J. Comp. Neurol. 158: 295-306.

Hubel, D. H., T. N. Wiesel, and D. M. -K. Lam (1974a) Autoradiographic demonstration of ocular-dominance columns in the monkey striate cortex by means of transneuronal transport. Brain Res. 79: 273-279.

Hubel, D. H., T. N. Wiesel, and S. LeVay (1974b) Visual field representation in layer IV $\mathrm{C}$ of monkey striate cortex. In Society for Neuroscience Program and Abstracts, 1974, p. 264, Society for Neuroscience, Bethesda, MD.

Hubel, D. H., T. N. Wiesel, and S. LeVay (1977) Plasticity of ocular dominance columns in monkey striate cortex. Philos. Trans. R. Soc. Lond. (Biol.) 278: 377-409.

Kennedy, C., M. H. Des Rosiers, J. W. Jehle, M. Reivich, F. Sharpe, and L. Sokoloff (1975) Mapping of functional neural pathways by autoradiographic survey of local metabolic rate with $\left[{ }^{14} \mathrm{C}\right]$ deoxyglucose. Science 187: 850-853.

LeVay, S. and M. P. Stryker (1979) The development of ocular dominance columns in the cat. In Society for Neuroscience Symposia. Vol. 4: Aspects of Developmental Neurobiology, pp. 83-98, Society for Neuroscience, Bethesda, MD.

I.eVay, S., D. H. Hubel, and T. N. Wiesel (1975) The pattern of ocular dominance columns in macaque visual cortex revealed by a reduced silver stain. J. Comp. Neurol. 159; 559-576.

LeVay, S., M. P. Stryker, and C. J. Shatz (1978) Ocular dominance columns and their development in layer IV of the cat's visual cortex: A quantitative study. J. Comp. Neurol. 179: 223-244.

LeVay, S., T. N. Wiesel, and D. H. Hubel (1980) The development of ocular dominance columns in normal and visually deprived monkeys. J. Comp. Neurol. 191: 1-51.

Livingstone, M. S., and D. H. Hubel (1984) Anatomy and physiology of a color system in the primate visual cortex. I. Neurnsci. 4: 309 356.

Malpeli, J. G. and F. H. Baker (1975) The representation of the visual field in the lateral geniculate nucleus of Macaca mulatta. J. Comp. Neurol. 161: $569-594$.

Mastronarde, D. N. (1983) Correlated firing of cat retinal ganglion cells. I. Spontaneously active inputs to $x$ - and $y$-cells. J. Neurophysiol. 49: $303-324$.

Meyer, R. L. (1984) Tetrodotoxin blocks the formation of ocular dominance columns in goldfish. Science 218: 589-591.

Polyak, S. (1957) The Vertebrate Visual System, University of Chicago Press, Chicago.

Rakic, P. (1976) Prenatal genesis of connections subserving ocular dominance in the rhesus monkey. Nature 261: 467-471.

Rolls, E. T. and A. Cowey (1970) Topography of the retina and striate cortex and its relationship to visual acuity in rhesus monkeys and squirrel monkeys. Exp. Brain. Res. 10: 298-310.

Schwartz, E. L. (1980) Computational anatomy and functional architecture of striate cortex: A spatial mapping approach to perceptual coding. Vision Res. 20: 645-669.

Stone, J., and E. Johnston (1981) The topography of primate retina: A study of the human, bushbaby and new- and old-world monkeys. J. Comp. Neurol. 196: 205-223.

Stryker, M. P. (1981) Late segregation of geniculate afferents to the cat's visual cortex after recovery from binocular impulse blockade. Soc. Neurosci. Abstr. 7: 842 .

Swindale, N. V. (1980) A model for the formation of ocular dominance stripes. Proc. R. Soc. Lond. (Biol.) 208: 243-264.

Swindale, N. V., F. Vital-Durand, and C. Blakemore (1981) Recovery from monocular deprivation in the monkey. III. Reversal of anatomical effects in the visual cortex. Proc. R. Soc. Lond. (Biol.) 213: 435450 .

Tootell, R. B. H., M. S. Silverman, E. Switkes, and R. L. De Valois (1982) Deoxyglucose analysis of retinotopic organization in primate striate cortex. Science 1218: 902-904.

Van Buren, J. M. (1963) The Retinal Ganglion Cell Layer, Charles C Thomas Publishers, Springfield, IL.

Van Essen, D. C., and J. H. R. Maunsell (1980) Two-dimensional maps of the cerebral cortex. J. Comp. Neurol. 191: 255-281.

Van Essen, D. C., W. T. Newsome, and J. H. R. Maunsell (1984) The visual field representation in striate cortex of the macaque monkey: Asymmetries, anisotropies, and individual variability. Vision Res. 24: $429-448$.

von der Malsburg, C. (1979) Development of ocularity domains and growth behavior of axon terminals. Biol. Cybernet. 32: 49-62. 